\title{
Usefulness of facial masks against COVID-19 transmission - an experimental evidence
}

\author{
Tomasz R. Sosnowski, Marcn Odziomek, Agata Dorosz
}

\begin{abstract}
Faculty of Chemical and Process Engineering, Warsaw University of Technology, POLAND, Warsaw, Waryńskiego 1, E-mail: Tomasz.Sosnowski@pw.edu.pl
\end{abstract}

\begin{abstract}
The primary route of transmission of COVID-19 disease is associated with the inhalation of airborne SAR-CoV-2 coronavirus exhaled by infected persons. Therefore, the use of facemasks has been proposed as one of the basic means of protection. This study presents methods of the evaluation of the protective properties of the masks at various exhalation conditions. The preliminary results show that even high-quality masks (respirators) offer only a partial elimination of aerosol particles due to the tightness issues.
\end{abstract}

Keywords - air filtration, personal protective equipment, filtration facemasks, aerosol exhalation.

\section{Introduction}

COVID-19 pandemic changed our world in 2020-21. Social distancing, hand hygiene, and covering the face with air-filtering masks have become the main recommendations in preventing the spread of the disease [1]. The actual value of these recommendations has been disputed due to the lack of firm data that would justify them. However, despite the ongoing debate $[2,3]$, face coverings are mandatory in many countries as primary protection means against coronavirus transmission via the aerosol route. It is based on the evidence that asymptomatic and presymptomatic infected persons can exhale the virus. The pathogen in the aerosol form can be inhaled by bystanders leading to further infection [4]. Considering this scenario, filtering out the exhaled air by facemasks is equally important as the filtration of inhaled air. Air filtration during inhalation requires very efficient masks (respirators) since low-class facemasks do not offer enough protection. Respirators are expensive and non-comfortable (due to high resistance), so they are not commonly used. Instead, most of the population wears less sophisticated face covers, e.g., surgical masks or cloth masks. Despite their lower filtration efficiency during inhalation, they may have good barrier properties against exhalation of the virus in the form of droplets generated in the respiratory system [5]. The aim of this work is to test this function under realistic in vitro conditions.

\section{Experimental}

Five types of face masks have been used in the preliminary studies (Table 1). They have been tested using a manikin head adapted for fitting the masks and aerosol emission via either a mouth opening or nostrils (Fig. 1a)

Table 1. The parameters of facemasks used in the studies

\begin{tabular}{|c|c|}
\hline Designation & Description \\
\hline SM & surgical mask \\
\hline F3 & FFP3 mask (respirator) without valve \\
\hline F2f & FFP2 mask (respirator) with the exhalation valve in the front \\
\hline F2s & FFP2 mask (respirator) with the exhalation valve on the side \\
\hline CLM & Cloth mask \\
\hline
\end{tabular}



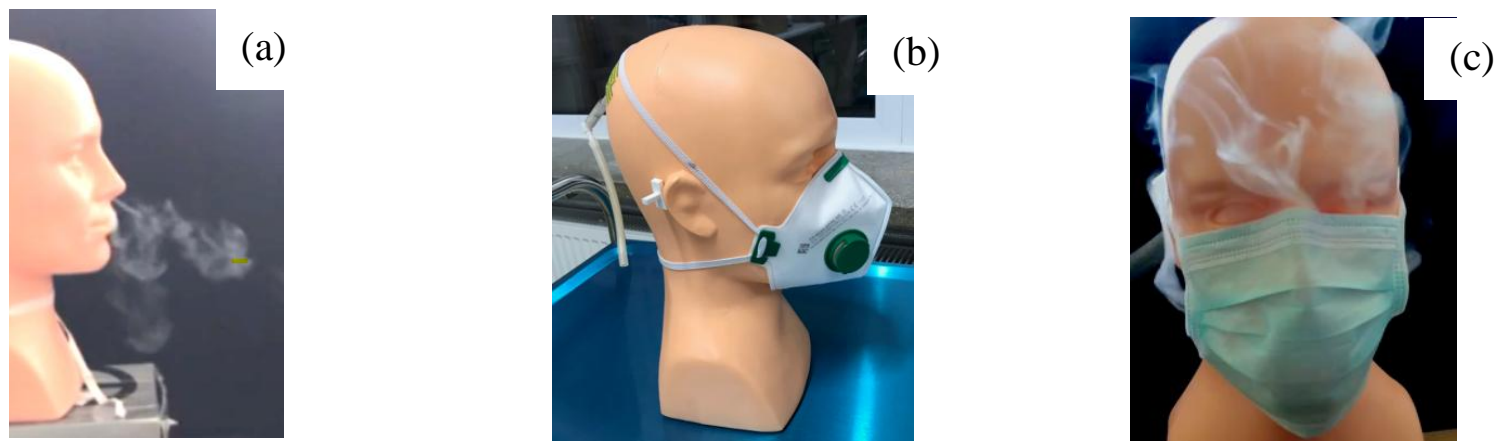

Fig.1. (a) Visualization of aerosol exhalation through the nostrils of the manikin; (b) the manikin wearing the F2vs mask; (c) visualization of aerosol penetration through the spaces of the inadequate fit of the surgical mask to the face surface.

Aerosol particles in the close vicinity of the manikin head were counted with the OPC-N3 laser spectrometer (Alphasense, Great Britain) that allowed to monitor the concentration of particles within the size range of $0.35-40 \mu \mathrm{m}$ which is representative for aerosols expelled from the respiratory system [5]. First trials with the wet aerosol generated from a medical nebulizer were unsuccessful due to the partial evaporation of droplets that made the results vague. Instead, we decided to generate the dry aerosol of gypsum powder using the pneumatic nebulizer (Sidestream, Philips Respironics, USA). This allowed suspending the aerosol in a 2 L glass chamber, from which the pulse of compressed air produced the puff of aerosol released through the manikin nostrils. Such a flow pattern was used to simulate realistic non-steady outflow conditions, that may be comparable, e.g., to a sneeze. The aerosol was blown into the particlefree atmosphere of purified sheath flow (Fig. 2), so the spectrometer could detect even a low number of particles penetrating the mask. The experimental setup (without the nebulizer) is schematically shown in Fig. 2. The airflow dynamics at the outflow from the manikin nostrils were determined in the independent measurement (with no aerosol in the system) with a digital airflow meter (model 3063, TSI Inc., USA).

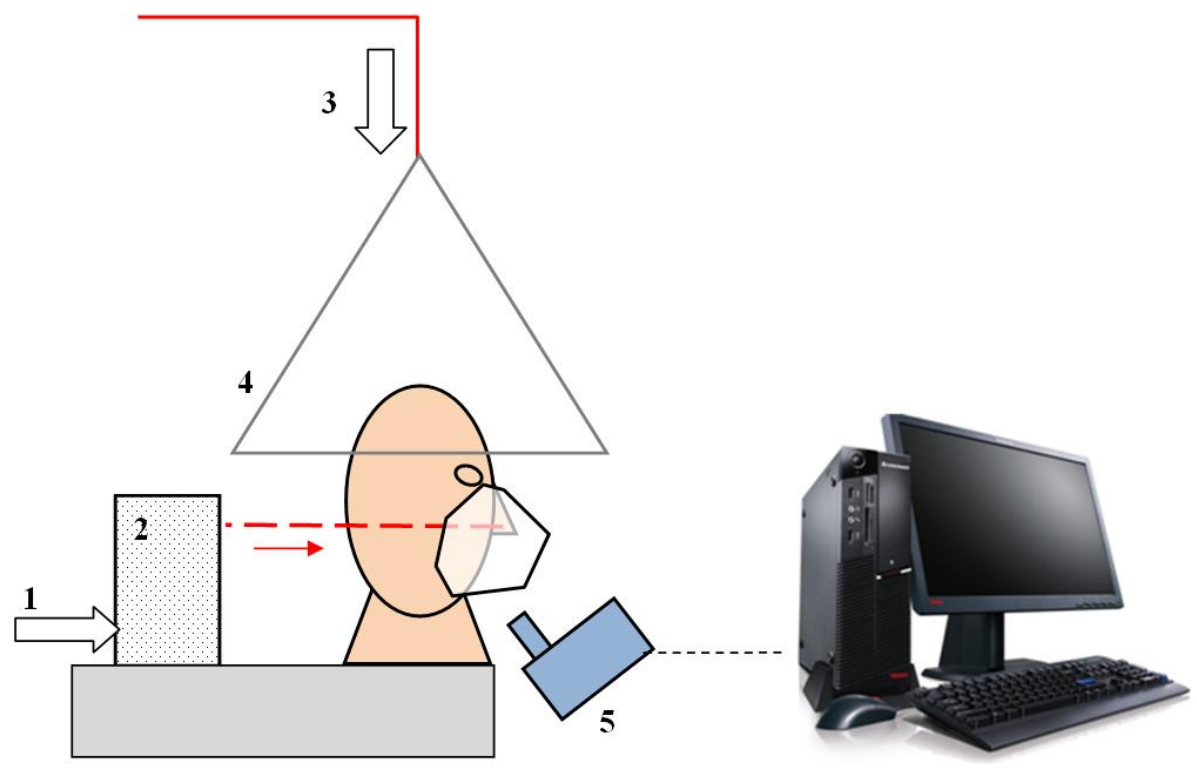

Fig.2. Experimental setup: 1 - pulse flows of the compressed air, 2 - aerosol chamber, 3 clean air (sheath flow), 4 - hood, 5 - aerosol spectrometer. 


\section{Results and discussion}

Fig. 3 shows the exhalation dynamics produced in the outflow of the manikin. It can be seen that the system allowed to generate short puffs with a duration of approximately $1.5 \mathrm{~s}$. They can be related to the cough or sneeze dynamics, although, in reality, the airflow rates are expected to be higher [6-8].

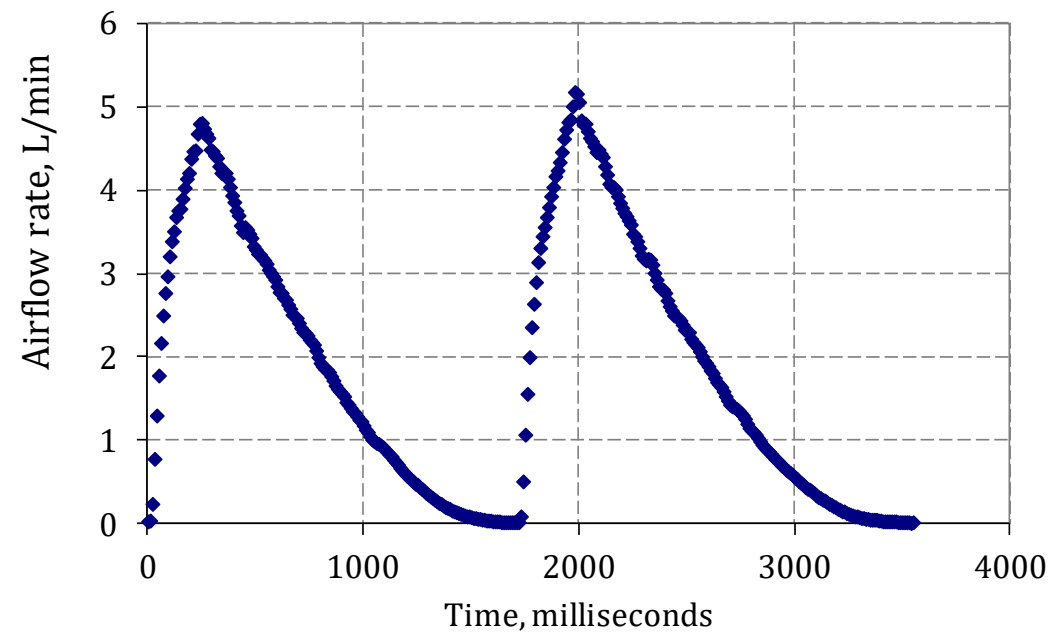

Fig.3. Air/aerosol flow dynamics generated in the system.

The overall efficiency of the five masks is compared in Fig.4. It is seen that even the most efficient F3 respirator allows for the escape of more than $40 \%$ of exhausted aerosol particles, whereas the results for less efficient facemasks are even worse. Interestingly, the side valve in the FFP2 mask (F2s) is responsible for a massive penetration of aerosol particles, which is comparable to the use of a simple surgical mask (SM). As expected, the masks (or respirators) designed mainly for the protection against inhaled aerosol particles, insufficiently filter out exhaled particles, especially under dynamic conditions. It can be attributed to a poor fitting of the mask to the face during exhalation, i.e., when the positive pressure builds up inside the mask.

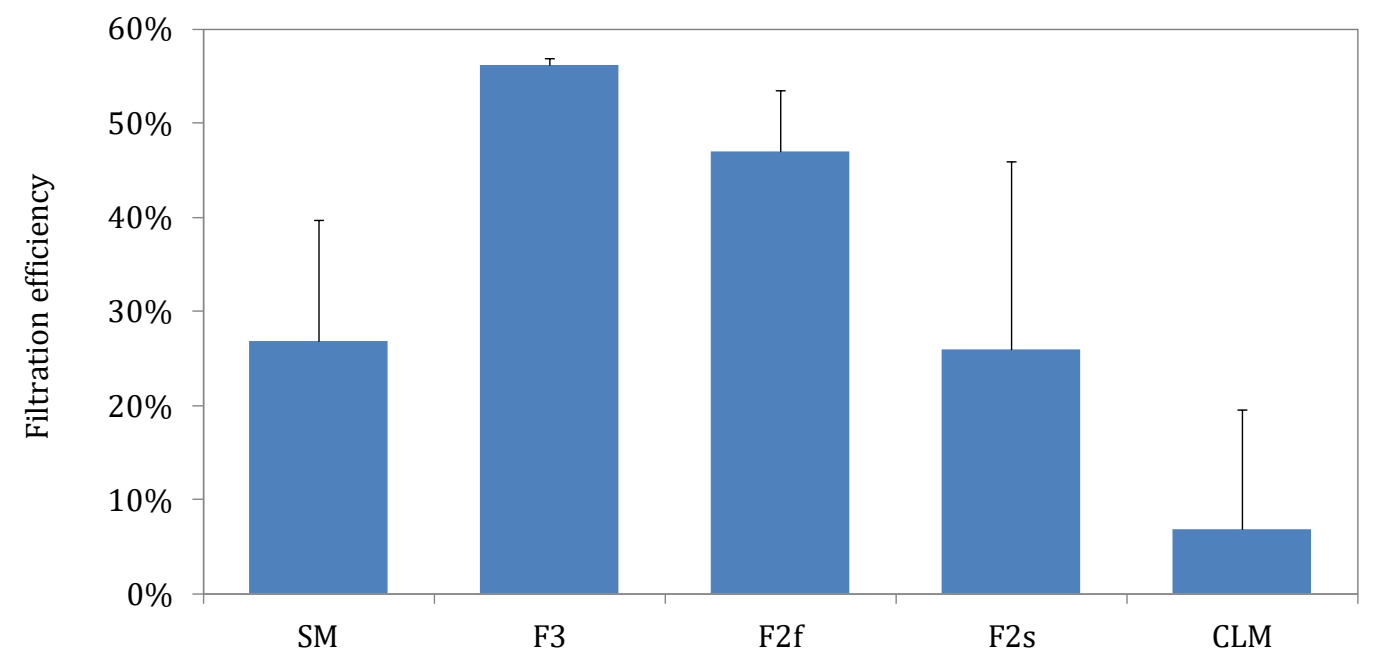

Fig.4. The efficiency of the respirators and masks regarding aerosol exhalation (error bars denote the standard deviation, $n=3$ ). 
Dynamic events such as cough or sneeze can push the mask out from the face and facilitate aerosol emission. Also, the valves (if present) increasing the breathing comfort, become open during exhalation and allow the particles to escape outside the mask.

Fig.5 shows the comparison of the particle size distribution in the aerosol penetrating the masks. The masks with a better filtration efficiency during exhalation remove mostly larger particles, so the cumulative size distribution is shifted towards smaller sizes comparing to the original aerosol ("no mask"). The mass median diameter values (d50) of the original and filtered aerosol are approximately 4 and $3 \mu \mathrm{m}$, respectively. The d90 (90 ${ }^{\text {th }}$ centile) is reduced from approximately 9 to $6 \mu \mathrm{m}$. These numbers suggest that the largest particles are eliminated, probably due to the predominating inertial mechanism of particle deposition on the inner surface of the facemasks.

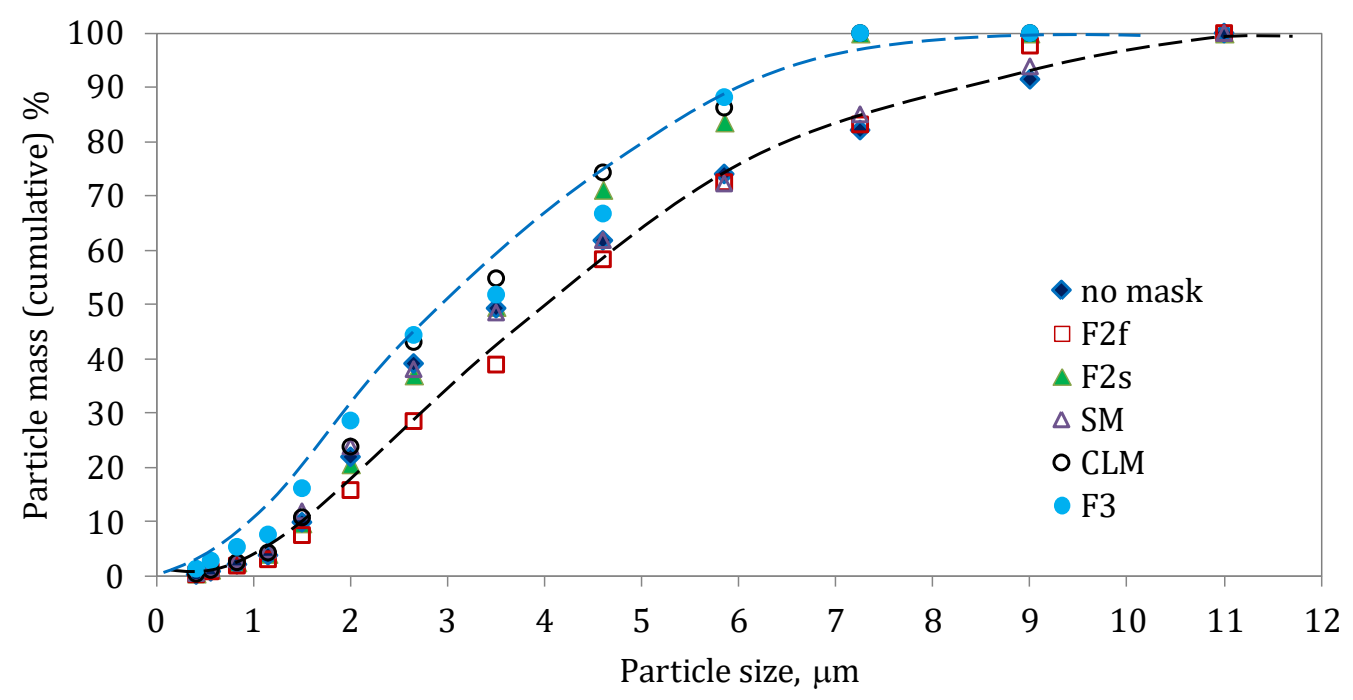

Fig.5. Cumulative mass-based particle size distribution measured beyond the facemasks.Two lines are drawn to visualize the shist of the distribution toward smaller size.

Based on the above results, it may be questioned if the use of facemasks can protect bystanders from the emission of coronavirus present in aerosol droplets exhaled by an infected individual. Let us notice two essential effects that lower the probability of infection in such a situation. The first one is related to the reduction of the number of aerosol particles that may contain the virus. The lower mass or volume median diameter indicates that the number of viruses potentially present in the aerosol suspended in the air is notably decreased (droplet volume is proportional to the third power of droplet size). It is known that the effective infection with SARS-CoV-2 requires many copies penetrating the respiratory system [9], so a reduction in the aerosol concentration matters.

The second factor that should be important for the usefulness of face covers, masks or respirators, is a deflection of the exhaled aerosol cloud, as seen, e.g., in Fig 1c. The droplets are directed not to the front but rather downwards, upwards, or to the sides, reducing the direct exposure of another person located head-to-head, e.g., in a group of people or during a conversation.

One of the limitations of this study is the application of a simplified model of the human head that does not necessarily reflect all essential geometrical features. In the near future we plan to use a standard head geometry according to the ISO/TS 16976-2:2015, which should provide a 
more realistic fit of the masks to the face. We also plan to continue experiments with the newly designed, dedicated cough and sneeze airflow generator, which should provide a more realistic representation of the loss of mask tightness during such events.

\section{Conclusion}

In vitro experiments with a model human head showed that facemasks and respirators do not fully protect the surrounding from aerosol particles emitted from the respiratory tract during flow pulses that correspond to exhalation or sneeze. The main reason is an adequate tightness of masks in the case of outward airflows. Nevertheless, it was demonstrated that masks reduce the concentration of emitted aerosol droplets, change their size distribution by filtering out the largest ones, and deflect the emitted aerosol cloud, so they act as a partial barrier and can reduce the chance of SARS-CoV-2 transmission.

\section{Acknowledgments}

Work supported by "IDUB against COVID" project grant by Warsaw University of Technology under the program Excellence Inititiave: Research University (IDUB).

\section{References}

[1] World Health Organization (2020). Mask use in the context of COVID-19. Interim report, December 2020, https://apps.who.int/iris/handle/10665/337199

[2] Mitze, T., Kosfeld, R., Rode, J., Wälde, K. (2020). Face masks considerably reduce COVID19 cases in Germany. PNAS 117(51), 32293-32301, https://doi.org/10.1073/ pnas. 2015954117

[3] Vainshelboim, B. (2021). Facemasks in the COVID-19 era: A health hypothesis. Medical Hypotheses, 146, 110411. https://doi.org/10.1016/j.mehy.2020.110411.

[4] Johansson, M. A., Quandelacy, T. M., Kada S., et al. (2021). SARS-CoV-2 transmission from people without COVID-19 symptoms. JAMA Network Open 4(1), e2035057, https://doi.org/10.1001/jamanetworkopen.2020.35057

[5] Sosnowski, T. R. (2021). Inhaled aerosols: their role in COVID-19 transmission including biophysical interactions in the lungs. Curr. Opin. Coll. Interface Sci. 54, 101451, https://doi.org/10.1016/j.cocis.2021.101451

[6] Bourouiba L. (2020). Turbulent gas clouds and respiratory pathogen emissions potential implications for reducing transmission of COVID-19. J. Am. Med. Assoc. 323 (18), 18371838, https://doi.org/10.1001/jama.2020.4756

[7] Tang J. W., Nicolle A.D., Klettner C.A., et al. (2013). Airflow dynamics of human jets: sneezing and breathing - potential sources of infectious aerosols. PloS One 8, e59970, https://doi.org/10.1371/journal.pone.0059970

[8] Lindsley W. G, Reynolds J.S., Szalajda J.V., Noti J.D., Beezhold D. H. (2013). A cough aerosol simulator for the study of disease transmission by human cough-generated aerosols, Aerosol Sci. Technol., 47(8), 937-944, https://doi.org/10.1080/02786826. 2013.803019

[9] Scheuch, G (2020). Breathing is enough: for the spread of influenza virus and SARS-CoV-2 by breathing only. J. Aerosol Med. Pulm. Drug. Deliv. 33, 230-234, https://doi.org/ 10.1089/jamp.2020.1616 